\title{
Factors associated with empathy among community pharmacists in Lebanon
}

\author{
Eva Hobeika ${ }^{1 *+}$, Souheil Hallit ${ }^{2,3^{*+}}$ (D) Hala Sacre ${ }^{2,4}$, Sahar Obeid ${ }^{1,2,5}$, Aline Hajj ${ }^{6,7}$ and Pascale Salameh ${ }^{2,8,9}$
}

\begin{abstract}
Introduction: Empathy is the cornerstone of the relationship between the healthcare provider and the patient. In Lebanon, no studies have investigated the factors associated with empathy among community pharmacists. Hence, the importance of this research to better understand empathy and help community pharmacists with this vital aspect of their practice.
\end{abstract}

Objective: This study aimed to evaluate empathy and possible factors associated with it among Lebanese community pharmacists.

Methods: This cross-sectional study was carried out between March and July 2018. It enrolled a proportionate random sample of 435 community pharmacists from all Lebanese districts. The Epi info software calculated the minimum sample size, based on a total number of 3762 community pharmacists, with an expected frequency of $50 \%$ of pharmacists with low empathy, and a 95\% confidence interval. The minimal sample size required was 350 community pharmacists; our sample size was 435 to account for missing values.

Results: Our results revealed that 228 (53.4\%) pharmacists had low empathy. Lower empathy was significantly associated with more physical (Beta $=-0.331)$ and mental $(B e t a=-0.126)$ work fatigue, higher age $($ Beta $=-0.125)$ and a practice experience between 3 years and less than 6 years compared to less than 6 months (Beta $=-2.440$ ).

Conclusion: This study shed the light on some factors associated with empathy among Lebanese community pharmacists. Low empathy levels were significantly associated with factors such as age, practice experience, and mental and physical work fatigue, all of which impact the practice, as the accepted model of pharmacy practice requires that pharmacists establish effective communication and use interpersonal skills. Therefore, developing empathetic communication skills is considered essential. Furthermore, increased mental and physical work fatigue should not hinder community pharmacists' access to self-care, whether for their mental or physical health.

Keywords: Empathy, Community pharmacists, Work fatigue, Lebanon

\section{Introduction}

Empathy is an ambiguous concept [1] that has been portrayed as a notion hard to define and measure [2]. Multiple definitions exist, but the one adapted for the patient

\footnotetext{
* Correspondence: eva.hobeika@hotmail.com; souheilhallit@hotmail.com ${ }^{\dagger}$ Eva Hobeika and Souheil Hallit are first co authors and contributed equally to this work.

${ }^{1}$ Faculty of Arts and Sciences, Holy Spirit University of Kaslik (USEK), Jounieh, Lebanon ${ }^{2}$ INSPECT-LB: Institut National de Santé Publique, Épidémiologie Clinique et Toxicologie, Beirut, Lebanon

Full list of author information is available at the end of the article
}

care context is: "predominantly a cognitive attribute that involves an understanding of patients' concerns, the capacity to communicate this understanding, and an intention to help" [1, 3]. It is divided into three components: affective or emotional, cognitive, and somatic. In this paper, empathy will refer to emotional empathy.

Previous studies have established that empathy is the cornerstone of the relationship between the healthcare provider and the patient $[1,4]$. Indeed, greater empathy was linked to better patient compliance [5, 6], more 
accurate diagnosis [7] and prognosis [8], and increased patient satisfaction [6]. Among the various categories of healthcare professionals, community pharmacists are considered to be the most accessible ${ }^{5}$. Previous findings showed that pharmacists who can communicate empathetically build a good rapport with patients, thus improving patient outcomes [9].

Evidence showed a negative association between empathy and burnout, higher empathy being associated with lower levels of burnout $[10,11]$, noting that burnout includes mental exhaustion, negative attitudes, and physical depletion. Moreover, the increased psychological distress among healthcare providers is related to decreased empathy and, consequently, alters the quality of care provided [12]. Furthermore, higher depressive symptoms have been correlated with lower empathy, suggesting that efforts to reduce depression may improve levels of empathy [13]. Also, good quality of sleep and recreational activities and exercise were also associated with higher empathy [14].

On the other hand, the relationship between empathy and gender showed that women had higher empathy levels compared to men, to a nearly significant degree [15, 16]. Although empathy is essential for patient care, it declines as medical students progress through training [17]. However, evaluations of the relationship between age and empathy among healthcare professionals, reveal that older practitioners have a higher level of empathy, mainly attributed to the maturity acquired over the years [18].

In 1994, Mark H. Davis introduced a social psychological approach to empathy. It suggested that the individual's empathic abilities can plausibly influence their management of conflict or other relationship-related behaviors. To inclusively define empathy, Davis proposed an organizational model that would take into account the different constructs falling under the broad heading of "empathy" [19]. This organizational model breaks down a typical empathy encounter into an exposure of the observer to the target, followed by a cognitive, affective, and/or behavioral response taking place from the observer's side [19]. Four related constructs make up the skeletal structure of this model. Antecedents, processes, intrapersonal, and interpersonal outcomes are delineated by associations between them; especially with constructs adjacent to each other. Davis' model (1994) has been used extensively as the underlying theoretical framework [20], in healthcare-related literature, aiming at understanding the organizational approach to empathy between healthcare providers and patients. It opened the door towards a better understanding of empathetic interactions in the workplace. Moreover, Gerace et al. integrated themes into Davis' linear model of empathy, delineating nurses' efforts for empathic communication with patients [20]. This study has also added a double-headed arrow to indicate perspective-taking as a means to regulate nurses' emotions towards patients (not present in the original model) [20]. Other scales have been used extensively among health professionals, such as the Jefferson Scale of Empathy. However, it does not offer theoretical tools necessary to establish a textbook basis for the works of empathy, as compared to Davis' model that provides an organizational aspect to the empathic interactions between healthcare providers and patients.

Lebanon is a country with a political instability, especially after the displacement of over a million Syrian refugees since 2012, which had a negative impact on the country economically and socially $[21,22]$ and created higher xenophobic attitudes among Lebanese in general [23]. Furthermore, community pharmacists are not satisfied financially especially after the drop in the medications prices following the decisions taken by the Ministry of Public Health [23], which added more stressors to their daily life and a lower quality of life [24].

Based on Davis and Gerace models, Fig. 1 presents an adapted version of the organizational model shedding the light on empathy among community pharmacists. In this figure, the antecedents construct enumerates several factors that community pharmacists have brought to the situation. These factors have the potential to influence both processes and outcomes towards empathy [19] and will be assessed throughout our study.

To the best of our knowledge, no studies have investigated the factors associated with empathy among community pharmacists. Hence, the importance of this research to better understand empathy and help community pharmacists with this vital aspect of their practice.

Therefore, this study aimed to evaluate empathy and possible factors associated with it among Lebanese community pharmacists.

\section{Methods \\ General study design}

This cross-sectional study was carried out between March and July 2018. It enrolled a proportionate random sample of 435 community pharmacists from all five Lebanese Mohafazat. Geographically, Lebanon is divided into five major districts, termed Mohafazat, Beirut, North, Mount Lebanon, Beqaa, and South. This sample is based on an exhaustive list provided by the Lebanese Order of Pharmacists (OPL, the official association of pharmacists in Lebanon). The methodology used is described elsewhere [24, 25].

\section{Sample size calculation}

According to the OPL list, a total of 3762 community pharmacists (employers and employees) practice in 3157 community pharmacies distributed across all regions. 


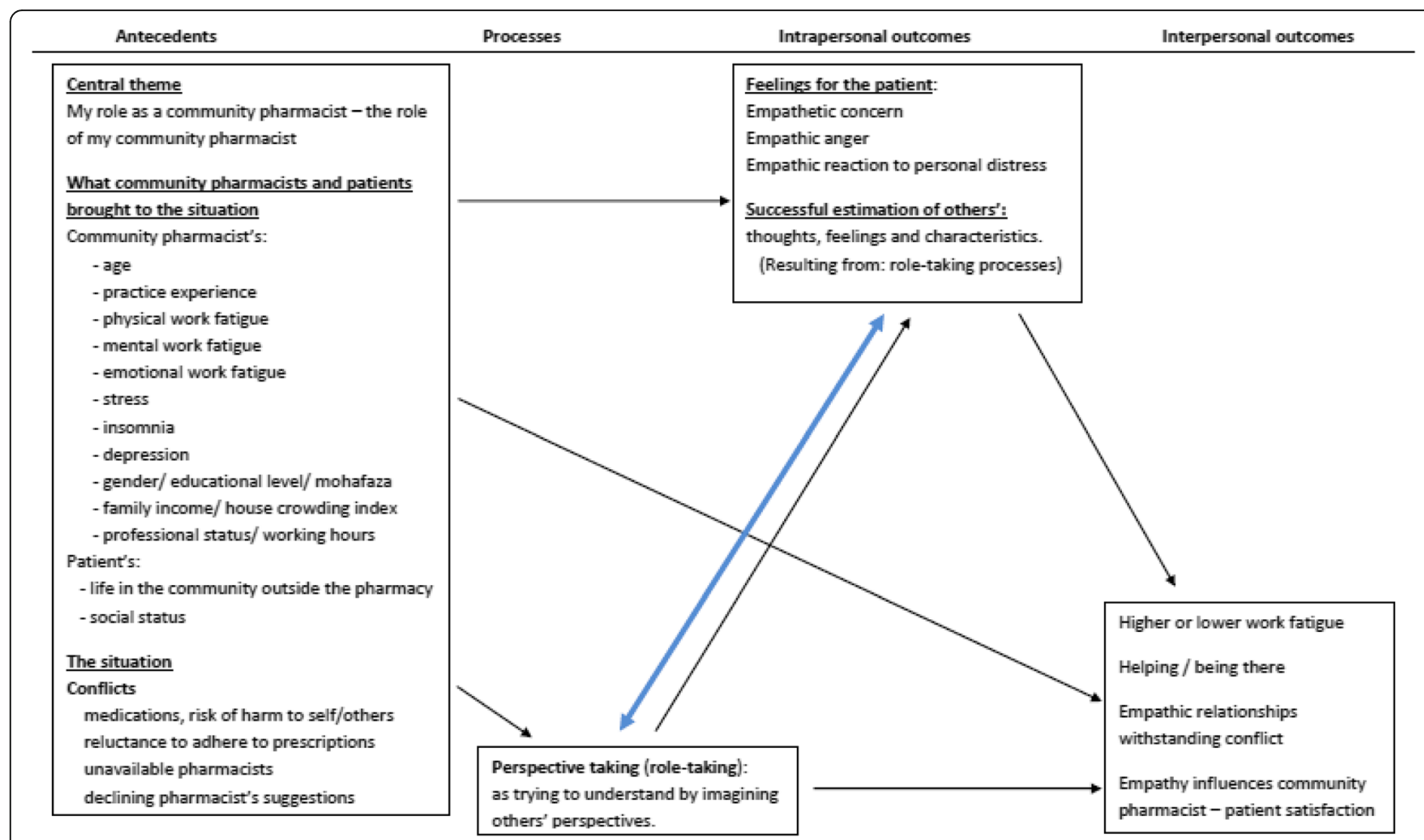

Fig. 1 The Organization Model of Empathy in Community Pharmacists. Framework built using Davis's (1994) linear antecedents, processes, intrapersonal and interpersonal outcomes framework [19]

The Epi info software calculated the minimum sample size, based on a total number of 3762 community pharmacists, with an expected frequency of $50 \%$ of pharmacists with low empathy (in the absence of since similar studies in the country), and a 95\% confidence interval. The minimal sample size required was 350 community pharmacists.

Out of the 500 randomly distributed questionnaires (in 500 pharmacies), 435 (87\%) were completed and collected back; the remaining 65 (13\%) corresponded to pharmacists who refused to participate in this study. Thus, the total sample included 435 participants.

\section{Questionnaire and variables}

The questionnaire was self-administered, closed-ended, and available in either French or English, the teaching languages in Lebanese schools of pharmacy. Welltrained field workers distributed it to the pharmacists after explaining the study objectives and obtaining written informed consent. To ensure optimal objectivity, pharmacists filled out the questionnaire without getting any guidance on any of the questions. The average completion time was between 15 and 20 min. Field workers collected back the questionnaires and sent them in closed boxes for data entry. This process allowed to preserve pharmacists' anonymity.
The questionnaire consisted of two sections. The first section included socio-demographic and practice characteristics, i.e., age, gender, demographic area, level of education, years of practice, location of the pharmacy, the approximate number of patients per day, job position, working hours per week. A house crowding index was also assessed. It was calculated by dividing the total number of individuals living in the house by the total number of rooms, excluding bathrooms and kitchen.

The second section included the following scales:

\section{Toronto empathy questionnaire}

It consists of 16 items [26]. A five-point scale, ranging between "never" and "often", is used to rate each item. Positively worded items $1,3,5,6,8,9,13$, and 16 were scored as: 0 (Never); 1 (Rarely); 2 (Sometimes); 3 (Often); 4 (Always), whereas negatively worded items 2 , $4,7,10,11,12,14$ and 15 were reversed. All the scores were summed to derive the total empathy score. Higher scores designated higher empathy. The Cronbach's alpha in this study was 0.729 .

\section{The three-dimensional work fatigue inventory (3D-WFI)}

This inventory consists of 18 questions divided into three 6-question packs. Each pack measured one dimension of work fatigue: physical (e.g., feeling physical exhaustion at the end of the workday), mental (e.g., facing 
difficulty to think and concentrate at the end of the workday), and emotional (e.g., facing difficulty to show and deal with emotions at the end of the workday) [27]. The score ranged from 0 (never) to 4 (every day). Higher scores indicated higher fatigue in all three dimensions. The Cronbach's alpha values were 0.880 (physical work fatigue), 0.710 (mental work fatigue), and 0.848 (emotional work fatigue).

\section{Hamilton depression rating scale (HDRS)}

This 17-item scale is validated in Lebanon [28]. It measures the severity of depressive symptoms (e.g., feelings of guilt, depressed mood, suicide, etc.) [29]. The total score is computed by summing the answers to the 17 questions, with higher scores indicating higher levels of depression. The Cronbach's alpha value was 0.870 for this study.

\section{Beirut distress scale (BDS-22 scale)}

This 22-item scale, validated in Lebanon, is used to screen for stress [30]. It assesses six factors in adults, over the past week: demotivation, depressive symptoms, psychosomatic symptoms, mood deterioration, intellectual inhibition, and anxiety. The total score is calculated on a 4-point Likert scale from 0 (not at all) to 3 (all of the time), with higher scores indicating higher levels of stress. In this study, the Cronbach's alpha was of 0.935 .

\section{Lebanese insomnia scale (LIS-18)}

This scale, validated in Lebanon, is used to screen for insomnia [31]. It consists of 18 items scored on a 5-point Likert scale from 1 (never) to 5 (always). Items 4,18 , and 22 are reversed. Higher scores indicate higher insomnia. In this study, the Cronbach's alpha was 0.811 .

\section{Forward and back translation procedure}

The translation from English into French was carried out by a translator and validated by a healthcare professionals' expert committee, and a language professional. A backward translation was then performed by a native English-speaking translator, fluent in French and unfamiliar with the notions of the scales. The expert committee compared the back-translated English version to the original one and resolved discrepancies and inconsistencies by consensus. Both versions were piloted on a sample of 20 pharmacists, before launching data collection. The results of the pilot sample were excluded from the final datasheet.

\section{Statistical analysis}

A study-independent person, not involved in the data collection process, performed the data entry. The statistical analysis was done on SPSS version 23. Student ttest was applied to check for associations between the empathy score and dichotomous variables (i.e., gender). The ANOVA test was used to compare means of 3 or more groups (i.e., educational level). Finally, a stepwise linear regression was performed, taking the self-reported empathy score as the dependent variable, and all the variables that showed a significant association in the bivariate analysis as independent variables. A $p$-value was significant when $p<0.05$.

\section{Results}

Sociodemographic characteristics of the participants and other parameters

Out of 500 distributed questionnaires, 435 (87\%) were completed and collected back. The mean age of the participants was $38.97 \pm 11.13$ years, and $52 \%$ were males (Table 1).

Since the Toronto empathy scale does not have a cutoff point, the median $(=60)$ was used as the cut-off point. The mean empathy score was $59.02 \pm 7.32$, with 228 (53.4\%) of the pharmacists having low empathy. Table 2 summarizes the scores of the intended measured parameters.

\section{Bivariate analysis}

The results of the bivariate analysis related to the factors associated with the empathy score are summarized in Tables 3 and 4 . The mean empathy score was slightly higher in females compared to males (59.95 vs. 58.17; $p=0.013)$. The mean empathy score was significantly higher in those having a Pharm.D degree (59.94) versus all other education levels, in those living in South Lebanon (61.41) compared to all other districts, and in those having a practice experience of fewer than 6 months. Lower empathy was significantly associated with older age $(r=-0.135)$, higher mental $(r=-0.401)$ and physical $(r=-0.399)$ work fatigue, higher insomnia $(r=$ - 0.098), and higher stress $(r=-0.233)$.

\section{Multivariable analysis}

The results of the stepwise linear regression, taking the empathy score as the dependent variable, revealed that lower empathy was significantly associated with more physical work fatigue (Beta $=-0.331)$, more mental work fatigue (Beta $=-0.126$ ), a practice experience between 3 years and fewer than 6 years compared to fewer than 6 months $($ Beta $=-2.440)$, and older age $($ Beta $=-0.125)$ (Table 5).

\section{Discussion}

A better understanding of empathy leads to a clearer comprehension of the patient-pharmacist interaction and, therefore, to a constructive relationship. The results of the multivariable analysis demonstrated that lower empathy was significantly associated with older age, 
Table 1 Sociodemographic and socioeconomic characteristics of the participants $(n=435)$

\begin{tabular}{|c|c|c|c|}
\hline Factor & N (\%) & Factor & N (\%) \\
\hline Gender & & > 3000 USD & $140(35.5 \%)$ \\
\hline Male & $223(52.0 \%)$ & Mean \pm SD & \\
\hline Female & $206(48.0 \%)$ & Age (in years) & $38.97 \pm 11.13$ \\
\hline District & & House crowding index & $0.89 \pm 0.44$ \\
\hline
\end{tabular}

more physical and mental work fatigue, and a practice experience between 3 years to fewer than 6 years compared to fewer than 6 months.

Our results showed that $53.4 \%$ of the Lebanese community pharmacists have low empathy, consistent with those found among American community pharmacists (58\% low empathy) [9]. Professional interactions are usually based on objectivity and ethical standards. Hence, the blurring of the line becomes understandable in professions that involve a certain degree of care-giving. Healthcare providers, such as pharmacists, get to a point where they either get too carried out by patients' concerns, or too little (low empathy). To reach an interactive balance, where empathy is not surpassed by either sympathy or lack of care for patients, pharmacists must learn to manage their empathetic behaviors [19].

In the present study, higher physical and mental work fatigue were associated with lower empathy, in agreement with results from previous research showing that community pharmacists with emotional exhaustion, depersonalization, and average job levels had mental disengagement, and reduced their commitment to helping patients [32-34]. Lebanese community pharmacists, although held in high esteem by the population [35] have a low level of job satisfaction due to financial constraints [36]. This dissatisfaction can explain the fact that pharmacists feel less concerned about communicating with patients and show less empathy towards them. Since the literature establishes a connection between financial constraints and lower empathy, it was used here to hypothesize an explanation for physical and mental work fatigue mediated by financial constraints.

Table 2 Description of the intended measured parameters in our sample

\begin{tabular}{ll}
\hline Variables & Mean \pm SD \\
\hline Empathy score & $59.02 \pm 7.32$ \\
Stress score & $42.37 \pm 13.49$ \\
Insomnia score & $37.53 \pm 8.44$ \\
Depression score & $6.90 \pm 7.01$ \\
Emotional work fatigue score & $17.38 \pm 10.42$ \\
Mental work fatigue score & $8.36 \pm 6.50$ \\
Physical work fatigue score & $7.63 \pm 8.29$ \\
\hline
\end{tabular}


Table 3 Bivariate analysis of factors associated with the empathy score

\begin{tabular}{|c|c|}
\hline Variable & Mean Empathy Score \\
\hline \multicolumn{2}{|l|}{ Gender } \\
\hline Male & $58.17 \pm 6.93$ \\
\hline Female & $59.95 \pm 7.61$ \\
\hline p-value & 0.013 \\
\hline \multicolumn{2}{|l|}{ Education level } \\
\hline Bachelor of Pharmacy & $59.36 \pm 6.83$ \\
\hline Pharm.D. & $59.94 \pm 7.65$ \\
\hline Master's degree & $56.96 \pm 7.78$ \\
\hline $\mathrm{PhD}$ & $56.50 \pm 7.30$ \\
\hline$p$-value & 0.038 \\
\hline \multicolumn{2}{|l|}{ District } \\
\hline Beirut & $56.85 \pm 7.58$ \\
\hline Mount Lebanon & $59.87 \pm 6.89$ \\
\hline North & $58.28 \pm 7.93$ \\
\hline South & $61.41 \pm 5.64$ \\
\hline Bekaa & $58.72 \pm 7.45$ \\
\hline p-value & 0.005 \\
\hline \multicolumn{2}{|l|}{ Years of practice } \\
\hline Less than 6 months & $63.16 \pm 4.87$ \\
\hline 6 months to less than 1 year & $62.15 \pm 8.73$ \\
\hline One year to less than 3 years & $59.25 \pm 8.84$ \\
\hline 3 years to less than 6 years & $57.85 \pm 7.32$ \\
\hline 6 years to less than 12 years & $59.46 \pm 7.69$ \\
\hline 12 years or more & $58.18 \pm 6.49$ \\
\hline P-value & 0.008 \\
\hline
\end{tabular}

Post hoc analysis: Districts (Beirut vs Mount Lebanon $p=0.033$; Beirut vs Bekaa $p=0.007$ ); Years of practice (less than 6 months vs 3 years to less than 6 years $p=0.034$; less than 6 months vs 12 years or more $p=0.025$ )

Our results showed that the third dimension of work fatigue, called emotional work fatigue, was not significantly associated with lower empathy. We speculate that emotional work fatigue is affected by several factors, i.e., pharmacist's state of mind, personality and other environmental cues that are not necessarily related to work performance. Thus, it is not directly affected by one source (the job at hand), like in the case of mental and physical work fatigue.

Previous findings [37] evaluating the correlation between empathy and age among pharmacists from all sectors (not just community pharmacists) have shown that older health professionals had greater empathy and were more assertive. Oppositely, our results revealed that older age was significantly associated with lower empathy; this difference can be explained by the job dissatisfaction experienced by the Lebanese community pharmacists [36]. Through continuous job dissatisfaction, pharmacists
Table 4 Bivariate analysis of continuous variables associated with the empathy score

\begin{tabular}{ll}
\hline Age & \\
\hline$r$ & -0.135 \\
$p$-value & $\mathbf{0 . 0 0 7}$ \\
Emotional work fatigue & \\
$r$ & -0.031 \\
$p$-value & 0.527 \\
Mental work fatigue & \\
$r$ & -0.401 \\
$p$-value & $<0.001$ \\
Physical work fatigue & \\
$r$ & -0.399 \\
$p$-value & $<\mathbf{0 . 0 0 1}$ \\
Stress score & \\
$r$ & -0.233 \\
p-value & $<0.001$ \\
Insomnia score & \\
$r$ & -0.098 \\
p-value & $\mathbf{0 . 0 4 3}$ \\
Depression score & \\
p-value & -0.077 \\
\hline
\end{tabular}

become less concerned and caring, expressing lower empathy levels towards their patients. As they get older, their relationship with loyal patients is affected by the lack of time taken to bond and empathize. So, this low level of empathy might affect the pharmacist-patient relationship and result in losing recurring customers over time, which in turn ends in job frustration because of a dissatisfactory income. Hence, the observed loop-cycle of older age leading to continuous job dissatisfaction, which leads to lower empathy.

Our study showed that having a practice experience between 3 years and less than 6 years was significantly associated with lower empathy. Previous studies [9] suggest that professional experience years play a role in the empathetic development process of community pharmacists: those more experienced develop empathetic skills socially, through a social learning model. Thus, pharmacists look up to fellow pharmacists or professional mentors during their formative professional years and use these observed attitudes and behaviors later on in their professional interactions [9]. Therefore, pharmacists having 3-6years of experience are in transition between learning from experienced mentors and developing their empathetic social skills. These pharmacists are in a middle zone, where they don't need mentors anymore but lack enough experience to navigate empathetic 
Table 5 Multivariable analysis: Linear regression taking the empathy score as the dependent variable

\begin{tabular}{|c|c|c|c|c|c|}
\hline Variable & Unstandardized Beta & Standardized Beta & $p$ & $95 \%$ Cor & ce Interval \\
\hline Physical work fatigue & -0.331 & -0.375 & $<0.001$ & -0.410 & -0.252 \\
\hline Age & -0.125 & -0.187 & $<0.001$ & -0.185 & -0.065 \\
\hline Mental work fatigue & -0.126 & -0.152 & 0.015 & -0.227 & 0.025 \\
\hline $\begin{array}{l}\text { Years of practice } 3 \text { years to less than } 6 \text { years compared to less } \\
\text { than } 6 \text { months compared to less than } 6 \text { months }^{\mathrm{a}}\end{array}$ & -2.440 & -0.122 & 0.009 & -4.275 & -0.605 \\
\hline
\end{tabular}

${ }^{\mathrm{a}}$ Reference group

interactions on their own. Being in this intermediate level can explain why they exhibit low empathy levels when compared to the other groups, keeping in mind that empathy is a social skill, mostly acquired through social practice and interactions with as many patients as possible through time. One way to improve empathy, in this case, is to develop continuous and integrated strategies to enhance empathic skills, and to progressively evaluate the ability of pharmacists to respond empathically throughout their clinical progression [38].

Statistically, our results found no significant relationship between depression score and empathy level. This may seem counterintuitive, but several well-cited studies explain the lack of association between different measures of empathy and depression. $\mathrm{O}^{\prime}$ Connor et al. [39] suggest that the ability to know what people are thinking, termed Theory of Mind (ToM), is a prerequisite for empathy and is common in depressed people. However, healthy empathy also requires an understanding of causality, which is affectively distorted in depression. Therefore, the presence of depression is not associated with empathy levels since depressed people may have normal cognitive empathy levels, but a different empathy experience (due to the misinterpretation of empathy).

\section{Clinical implications}

This study showed that lower levels of empathy were significantly associated with older age, increased physical and mental work fatigue, and practice experience between 3 years and less than 6 years. No future measures or adopted solutions can affect the age-related factor. However, it is possible to improve work fatigue conditions. One way to decrease fatigue is for pharmacists: to get proper rest, attend to the body's physiological needs by maintaining balanced lifestyles, ensure good physical health, and receive the support needed for proper mental health. Since empathy is an active dynamic process, the community pharmacist becomes more receptive to patients' needs after decreasing work fatigue. This way, the pharmacist is better equipped to reach out to patients and fulfill the accepted model of pharmacy practice, which requires effective communication and the use of interpersonal skills.
Pharmacy schools took many initiatives to include curriculum modifications, aiming at enhancing empathy in pharmacists during their training. Indeed, by going to the source as early as possible, pharmacy students get a solid formation on empathy and its psychosocial attributes [40]. Implementing different learning techniques i.e., simulated learning with ethical scenarios, interprofessional and problem-based learning, can lead to enhanced values, ethics, and decision-making [41]. Several studies show that empathetic relationships with patients are followed by rewards, with empathetic dialogue leading to patient satisfaction, adherence to treatment (sometimes), comprehension, and enhanced clinical outcomes [42].

\section{Limitations}

Our study has several limitations. First, information bias may occur since the questionnaire used is based on selfreport of pharmacists' thoughts and interpretation of symptoms. Social desirability bias may also be noted since participants may want to seem conforming to social norms; this was taken into consideration by making sure that they know that their answers remain anonymous. Secondly, no causality inferences can be made since this study is cross-sectional. Further longitudinal studies are warranted to make causality inferences. However, for this study, we were able to make associations (evaluation of factors associated with empathy). Thirdly, some of the scales used in the questionnaire, such as the Toronto Empathy Questionnaire, are not validated in Lebanon. Nevertheless, the results derived from these scales were found to be noteworthy by the authors, since they provide consistency upon comparison to other studies.

\section{Conclusion}

This study shed the light on some factors associated with empathy among Lebanese community pharmacists. Low empathy levels were significantly associated with factors such as age, practice experience, and mental and physical work fatigue, all of which impact the practice, as the accepted model of pharmacy practice requires that pharmacists establish effective communication and use interpersonal skills. Therefore, developing empathetic communication skills is considered essential. 
Furthermore, increased mental and physical work fatigue should not hinder community pharmacists' access to self-care, whether for their mental or physical health.

\section{Acknowledgments}

We would like to thank all pharmacists who participated in this study.

\section{Authors' contributions}

SH and PS designed the study; EH ad SH drafted the manuscript; SH and PS carried out the analysis and interpreted the results; $\mathrm{SO}$ and $\mathrm{AH}$ assisted in drafting and reviewing the manuscript; HS edited the paper for English language. All authors reviewed the final manuscript and gave their consent. The author(s) read and approved the final manuscript.

\section{Funding}

None.

\section{Ethics approval}

The study protocol was approved by the Psychiatric Hospital of the Cross's Ethics committee (HPC-006-2019). From each pharmacist, their written informed consent was acquired.

\section{Competing interests}

The authors have no conflicts of interest to disclose.

\section{Author details}

${ }^{1}$ Faculty of Arts and Sciences, Holy Spirit University of Kaslik (USEK), Jounieh, Lebanon. ${ }^{2}$ INSPECT-LB: Institut National de Santé Publique, Épidémiologie Clinique et Toxicologie, Beirut, Lebanon. ${ }^{3}$ Faculty of Medicine and Medical Sciences, Holy Spirit University of Kaslik (USEK), Jounieh, Lebanon. ${ }^{4}$ Drug Information Center, Order of Pharmacists of Lebanon, Beirut, Lebanon. ${ }^{5}$ Departments of Psychology and Research, Psychiatric Hospital of the Cross, Jal Eddib, Lebanon. 'Laboratory of Pharmacology, Clinical Pharmacy and Quality Control of Drugs, Faculty of Pharmacy, Pôle Technologie-Santé (PTS), Faculty of Pharmacy, Saint-Joseph University, Beirut 1107 2180, Lebanon. 'Faculty of Pharmacy, Saint-Joseph University, Beirut 1107 2180, Lebanon. ${ }^{8}$ Faculty of Pharmacy, Lebanese University, Hadat, Lebanon. ${ }^{9}$ Faculty of Medicine, Lebanese University, Hadat, Lebanon.

\section{Received: 27 March 2020 Accepted: 1 June 2020}

\section{Published online: 14 August 2020}

\section{References}

1. Hojat M. Empathy in patient care: antecedents, development, measurement, and outcomes: Springer Science \& Business Media; 2007. https:/psycnet. apa.org/record/2006-20612-000.

2. Kestenbaum R, Farber EA, Sroufe LA. Individual differences in empathy among preschoolers: relation to attachment history. New Dir Child Dev. 1989:44:51-64

3. Hojat M. Ten approaches for enhancing empathy in health and human services cultures. J Health Hum Serv Adm. 2009:31(4):412-50.

4. Reiss H. Empathy in medicine: a neurological perspective. J Am Med Assoc. 2010:304(14):1604-5.

5. DiMatteo MR, Sherbourne CD, Hays RD, Ordway L, Kravitz RL, McGlynn EA, et al. Physicians' characteristics influence patients' adherence to medical treatment: results from the medical outcomes study. Health Psychol. 1993; 12(2):93-102.

6. Kim SS, Kaplowitz S, Johnston MV. The effects of physician empathy on patient satisfaction and compliance. Eval Health Prof. 2004;27(3):237-51.

7. Barsky AJ. Hidden reasons some patients visit doctors. Ann Intern Med. 1981;94(4_Part_1):492-8.

8. Dubnicki C. Relationships among therapist empathy and authoritarianism and a therapist's prognosis. J Consult Clin Psychol. 1977;45(5):958-9.

9. Lonie JM. From counting and pouring to caring: the empathic developmental process of community pharmacists. Res Social Adm Pharm. 2006;2(4):439-57.

10. Brazeau CM, Schroeder R, Rovi S, Boyd L. Relationships between medical student burnout, empathy, and professionalism climate. Acad Med. 2010; 85(10 Suppl):S33-6.

11. Wilkinson $H$, Whittington $R$, Perry $L$, Eames $C$. Examining the relationship between burnout and empathy in healthcare professionals: a systematic review. Burn Res. 2017;6:18-29.
12. Bellini LM, Baime M, Shea JA. Variation of mood and empathy during internship. JAMA. 2002;287(23):3143-6.

13. Thomas MR, Dyrbye LN, Huntington JL, Lawson KL, Novotny PJ, Sloan JA, et al. How do distress and well-being relate to medical student empathy? A multicenter study. J Gen Intern Med. 2007;22(2):177-83.

14. Faye A, Kalra G, Swamy R, Shukla A, Subramanyam A, Kamath R. Study of emotional intelligence and empathy in medical postgraduates. Indian J Psychiatry. 2011;53(2):140-4.

15. Gleichgerrcht E, Decety J. Empathy in clinical practice: how individual dispositions, gender, and experience moderate empathic concern, burnout, and emotional distress in physicians. PLoS One. 2013;8(4):e61526.

16. Quince TA, Kinnersley $P$, Hales J, da Silva A, Moriarty $H$, Thiemann $P$, et al. Empathy among undergraduate medical students: a multi-Centre crosssectional comparison of students beginning and approaching the end of their course. BMC Med Educ. 2016:16:92.

17. Hojat M, Vergare MJ, Maxwell K, Brainard G, Herrine SK, Isenberg GA, et al. The devil is in the third year: a longitudinal study of erosion of empathy in medical school. Acad Med. 2009;84(9):1182-91.

18. Girdharwal N. Study on emotional intelligence at health care industry. San Mateo, C; 2007.

19. Davis MH. Empathy: a social psychological approach: Routledge; 2018

20. Gerace A, Oster C, O'kane D, Hayman CL, Muir-Cochrane E. Empathic processes during nurse-consumer conflict situations in psychiatric inpatient units: a qualitative study. Int J Ment Health Nurs. 2018;27(1):92-105.

21. Cherri Z, González PA, Delgado RC. The Lebanese-Syrian crisis: impact of influx of Syrian refugees to an already weak state. Risk Manage Healthcare Policy. 2016;9:165

22. Karam E, El Chammay R, Richa S, Naja W, Fayyad J, Ammar W. Lebanon: mental health system reform and the Syrian crisis. BJPsych Int. 2016;13(4): 87-9.

23. Obeid S, Haddad C, Salame W, Kheir N, Hallit S. Xenophobic attitudes, behaviors and coping strategies among Lebanese people toward immigrants and refugees. Perspect Psychiatr Care. 2019;55(4):710-7.

24. Sacre H, Obeid S, Choueiry G, Hobeika E, Farah R, Hajj A, Akel M, Hallit S, Salameh P. Factors associated with quality of life among community pharmacists in Lebanon: results of a cross-sectional study. Pharm Pract. 2019;17(4):1613. https://doi.org/10.18549/PharmPract.2019.4.1613.

25. Rahme D, Lahoud N, Sacre H, Akel M, Hallit S, Salameh P. Work fatigue among Lebanese community pharmacists: prevalence and correlates. Pharm Pract. 2020;18(2):1844. https://doi.org/10.18549/PharmPract.2020.2.1844.

26. Spreng RN, McKinnon MC, Mar RA, Levine B. The Toronto Empathy Questionnaire: Scale development and initial validation of a factor-analytic solution to multiple empathy measures. J Pers Assess. 2009;91(1):62-71.

27. Frone MR, Tidwell MO. The meaning and measurement of work fatigue: development and evaluation of the three-dimensional work fatigue inventory (3D-WFI). J Occup Health Psychol. 2015;20(3):273-88.

28. Obeid S, Abi Elias Hallit C, Haddad C, Hany Z, Hallit S. Validation of the Hamilton Depression Rating Scale (HDRS) and sociodemographic factors associated with Lebanese depressed patients. Encephale. 2018;44(5):397402. https://doi.org/10.1016/j.encep.2017.10.010.

29. Hamilton M. A rating scale for depression. J Neurol Neurosurg Psychiatry. 1960;23:56-62.

30. Barbour B, Saadeh N, Salameh PR. Psychological distress in Lebanese young adults: constructing the screening tool 'BDS-22'. Int J Cult Ment Health. 2012:5(2):94-108.

31. Hallit S, Sacre H, Haddad C, et al. Development of the Lebanese insomnia scale (LIS-18): a new scale to assess insomnia in adult patients. BMC Psychiatry. 2019;19:421. https://doi.org/10.1186/s12888-019-2406-y.

32. Thomas PG. Burnout and work engagement levels in community pharmacists residing in three countries of the United Kingdom. England: University of Portsmouth; 2011.

33. Basson M, Rothmann S. Sense of coherence, coping and burnout of pharmacists. S Afr J Econ Manag Sci. 2002;5(1):35-62.

34. El Kettani A, Serhier Z, Othmani MB, Agoub M, Battas O. French language questionnaires assessing burnout among caregivers: a literature review. JMSR. 2019:6(1):622-6.

35. Iskandar K, Hallit S, Raad EB, Droubi F, Layoun N, Salameh P. Community pharmacy in Lebanon: A societal perspective. Pharm Pract (Granada). 2017; 15(2):893

36. Hallit S, Zeenny RM, Sili G, Salameh P. Situation analysis of community pharmacy owners in Lebanon. Pharm Pract (Granada). 2017;15(1):853. 
37. Burgon J, Hamerslag L. The relationship between scientific research, clinical trials and FDA drug approval. Value Health. 2013;16(7):A478.

38. Hastings TJ, Kavookjian J, Ekong G. Associations among student conflict management style and attitudes toward empathy. Curr Pharm Teach Learn. 2019;11(1):25-32

39. O'Connor LE, Berry JW, Lewis T, Mulherin K, Crisostomo PS. Empathy and depression: The moral system on overdrive. In T. \& Farrow P. Woodruff (Eds.). Empathy in mental illness. Cambridge University Press; 2007. p. 49-75. https:// doi.org/10.1017/CBO9780511543753.005.

40. Tamayo CA, Rizkalla MN, Henderson KK. Cognitive, Behavioral and Emotional Empathy in Pharmacy Students: Targeting Programs for Curriculum Modification. Front Pharmacol. 2016;7:96.

41. Rajiah K, Venaktaraman R. The effect of demographic and social factors on the decision-making of community pharmacists in ethical dilemmas. J Res Pharm Pract. 2019;8(3):174-7.

42. Ciemins EL, Brant J, Kersten D, Mullette E, Dickerson D. A qualitative analysis of patient and family perspectives of palliative care. J Palliat Med. 2015;18(3): 282-5.

\section{Publisher's Note}

Springer Nature remains neutral with regard to jurisdictional claims in published maps and institutional affiliations.

Ready to submit your research? Choose BMC and benefit from:

- fast, convenient online submission

- thorough peer review by experienced researchers in your field

- rapid publication on acceptance

- support for research data, including large and complex data types

- gold Open Access which fosters wider collaboration and increased citations

- maximum visibility for your research: over $100 \mathrm{M}$ website views per year

At $\mathrm{BMC}$, research is always in progress.

Learn more biomedcentral.com/submissions 